\title{
Transcranial Magnetic and Direct Current Stimulation in the Treatment of Depression: Basic Mechanisms and Challenges of Two Commonly Used Brain Stimulation Methods in Interventional Psychiatry
}

\author{
Aditya Singh $^{\mathrm{a}}$ Tracy Erwin-Grabner ${ }^{\mathrm{a}}$ Roberto Goya-Maldonado ${ }^{\mathrm{a}}$ \\ Andrea Antal ${ }^{b, c}$ \\ ${ }^{a}$ Laboratory of Systems Neuroscience and Imaging in Psychiatry (SNIP-Lab), Department of Psychiatry \\ and Psychotherapy, University Medical Center Göttingen, Göttingen, Germany; ${ }^{b}$ Department of Clinical \\ Neurophysiology, University Medical Center Göttingen, Göttingen, Germany; ' Institute for Medical Psychology, \\ Medical Faculty, Otto-v.-Guericke University Magdeburg, Magdeburg, Germany
}

\section{Keywords}

Repetitive transcranial magnetic stimulation .

Transcranial direct current stimulation - Brain stimulation ·

Neuromodulation · Psychiatry · Depression

\begin{abstract}
Noninvasive neuromodulation, including repetitive transcranial magnetic stimulation (rTMS) and direct current stimulation (tDCS), provides researchers and health care professionals with the ability to gain unique insights into brain functions and treat several neurological and psychiatric conditions. Undeniably, the number of published research and clinical papers on this topic is increasing exponentially. In parallel, several methodological and scientific caveats have emerged in the transcranial stimulation field; these include less robust and reliable effects as well as contradictory clinical findings. These inconsistencies are maybe due to the fact that research exploring the relationship between the methodological aspects and clinical efficacy of rTMS and tDCS is far from conclusive. Hence, additional work is needed to un-
\end{abstract}

karger@karger.com www.karger.com/nps

Karger" derstand the mechanisms underlying the effects of magnetic stimulation and low-intensity transcranial electrical stimulation (TES) in order to optimize dosing, methodological designs, and safety aspects.

C) 2019 S. Karger AG, Basel

\section{Introduction}

Transcranial neuromodulation driven by repetitive transcranial magnetic stimulation (rTMS) and transcranial direct current stimulation (tDCS) has been found to be a promising noninvasive treatment for a variety of neuropsychiatric conditions [1-8]. Therapeutic utility of these methods has been claimed for psychiatric conditions such as depression, acute mania, bipolar disorder, panic, hallucinations, obsessions/compulsions, schizophrenia, catatonia, posttraumatic stress disorder, and drug cravings; neurologic diseases such as Parkinson's disease, dystonia, tics, stuttering, tinnitus, spasticity, epilepsy; rehabilitation of aphasia or of hand function after 
stroke; and pain syndromes such as neuropathic pain, visceral pain, or migraines [7-9].

TMS offers potential for higher efficacy and a lower number of adverse effects relative to pharmacotherapy or electroconvulsive therapy. As a result, the importance of this technique and the therapeutic possibilities are exponentially increasing. The most successful example has been the treatment of major depressive disorder (MDD), which resulted in several countries approving its use in clinical settings. The most frequently applied evidencebased treatment approach for MDD is high-frequency $(10 \mathrm{~Hz})$ rTMS over the left dorsolateral prefrontal cortex (DLPFC). It has a level A recommendation in the guidelines for good clinical practice, especially for the acute phase of treatment-resistant depression [9].

Similarly, with regard to the application of tDCS for MDD, the current approach is to enhance neural activity in the left DLPFC with anodal stimulation and/or to reduce neural activity in the right DLPFC with cathodal stimulation $[10,11]$. tDCS is recognized with a level B of evidence regarding the antidepressant efficacy of anodal tDCS of the left DLPFC in the guidelines for good clinical practice [8].

In spite of the therapeutic success in MDD, there are still several open questions with respect to the general clinical use of these methods in interventional psychiatry. Studies based on knowledge of rTMS effects, usually in the motor area, have led to further research and, consequentially, guidelines for using rTMS protocols in therapeutic practice $[9,12]$. However, many unanswered questions persist regarding how these differences in parameters of rTMS protocol might impact treatment efficacy and what are the possible ways forward to reduce such variations for maximal rTMS stimulation benefits. Currently, rTMS and tDCS are usually applied either as a monotherapy or as augmentation to pharmacotherapy and/or psychotherapy. Although the response to these treatment options is better than sham results in randomized controlled trials (RCTs), a large proportion of patients do not respond. It is therefore imperative to seek optimization of the treatment protocol through personalization of brain stimulation. Furthermore, relating to clinical practice, there is good evidence for beneficial antidepressant effects of transcranial stimulation, although to date the appropriate place of this technique in the therapeutic decision tree is still not clearly defined.

In this short review, we briefly outline the basic principles of rTMS and tDCS. Additionally, we describe how rTMS influences functional connectivity (FC) in response to depression treatment and discuss the sources that in- troduce disparity in the response to stimulation. Finally, we suggest ways to overcome some of these disparities. Applying these is crucial to boost the performance of rTMS in the treatment of depression.

\section{Transcranial Magnetic Stimulation}

TMS was first proposed as a method of brain stimulation in 1985 [13]. It facilitates not only relatively focal stimulation of cortical targets, but also more diffuse stimulation of larger brain volumes, thus penetrating deeper brain regions than $\mathrm{tDCS}$ can [14]. The basic principle behind TMS remains the same across protocols and works on Faraday's principle, i.e., a changing electric field induces a changing magnetic field of a few tesla, which in turn induces a perpendicular electric current in conductors in the near vicinity, i.e., a population of neurons [15]. There are several adjustable parameters when designing TMS protocols, such as the number of pulses, frequency, train length, and intertrain interval (ITI) [16]. The research has explored TMS protocols with variations of these parameters mostly on motor-evoked potentials (MEPs) and resting motor threshold (RMT) [17].

While single pulses can evoke MEPs that result in muscle responses when targeted at the primary motor cortex (M1), they are usually not enough to induce longer-lasting effects. Hence, the single pulses of TMS are applied in succession, in prescribed repetitive patterns that allow sustained after effects from the stimulation [18], called rTMS. Therefore, the frequency at which the pulses are delivered and the duration for which they are applied [19] contribute to the variations seen in rTMS protocols that impact the after effects observed. Frequencies of rTMS delivery $\leq 1 \mathrm{~Hz}$ are called low-frequency stimulation and induce inhibitory effects; high-frequency stimulation is $\geq 5 \mathrm{~Hz}$, and induces excitatory effects in brain $[17,20]$. Another important factor that can impact the effects of rTMS includes the intensity relative to the RMT at which the stimulation is delivered. Previous work has shown that if highfrequency rTMS is delivered at intensities lower than the RMT it decreases cortical excitability, while if delivered above the RMT it causes an increase in cortical excitability [21]. A recent study also showed that having adequate ITI during high-frequency rTMS may be essential to its efficacy, as the appropriate ITI can prevent the conduction failure of neurons and thus allow rTMS effects to carry through [22]. It has been proposed that the length of ITI may play a role in rTMS protocol efficacy and should be optimized for the disorder or a desired outcome. 
Combining rTMS with brain imaging has increased the understanding of rTMS effects and created the potential to dissect anatomical and functional cross-talk between different brain regions [16]. This ability to modulate brain activity and FC using rTMS has led to its use in clinical settings for therapeutic benefits [23], where it is employed to compensate for processes that are disturbed in psychiatric illnesses. For example, in MDD, rTMS over the DLPFC is used to compensate for deficient cortical excitability and FC [24].

Past research using positron emission tomography (PET) has shown left prefrontal glucose hypometabolism in patients with depression [25]. The use of excitatory rTMS as a treatment stemmed from the expectation of correcting for this hypoactivity in the frontal regions, as several groups had shown that the use of rTMS resulted in an increased blood flow in the prefrontal regions [26, 27] and increased activity under the TMS coil [27-29]. Studies have now gathered evidence for the clinical efficacy of high-frequency $(10 \mathrm{~Hz})$ rTMS $[30,31]$ to the left DLPFC, low-frequency $(1 \mathrm{~Hz})[32]$ to the right DLPFC, and bilateral rTMS treatment to both the left and the right DLPFC [33]. More recently, a large study established the noninferiority of intermittent theta-burst stimulation (iTBS) [34] over rTMS protocol. It showed that iTBS stimulation did not differ from $10-\mathrm{Hz}$ rTMS treatment in terms of dropout rates, expected side effects, safety, or tolerability as well as clinical benefits [35]. Thus, it has been established that rTMS (left high-frequency, right low-frequency, and bilateral) and iTBS are effective and reliable treatment options for depression, albeit with different degrees of response.

While PET studies have made a case for rTMS in the treatment of depression, other neuroimaging studies using simple and noninvasive techniques, such as restingstate functional magnetic resonance imaging (rsfMRI), have contributed important knowledge about FC aberrations and changes in depression cohorts compared to healthy populations. Studies have reported that depression patients consistently have dysfunctions associated with the default mode network (DMN), central executive network (CEN), and salience network (SN) [36-38]. Several studies have shed light on the importance of the subgenual anterior cingulate cortex (sgACC), an important node within the DMN, and its negative correlation to the stimulation site at the DLPFC for a better therapeutic response to rTMS [39-41]. Such results from studies using rsfMRI in subjects with depression have allowed for building a FC-based model of the disease. Utilizing these FC-based models, it is possible to stimulate such net-

Mechanisms and Challenges of Neuromodulation by tDCS and rTMS works to better understand the antidepressant mechanism of rTMS. For example, Liston et al. [37] reported on the effects of $10-\mathrm{Hz}$ rTMS on the functional networks in depressed subjects: higher sgACC-DMN connectivity in these subjects was decreased post-rTMS treatment, suggesting that rTMS acts by influencing sgACC-to-DMN connectivity. This is in line with the general implication of sgACC in depression treatment, where normalizing sgACC hyperactivity is associated with an antidepressant response [42, 43]. Another study [44] using iTBS for antidepressant treatment showed higher sgACC connectivity to the DLPFC and precuneus in individuals with depression, which normalized in response to rTMS treatment.

Numerous FC features have been employed to predict the response to rTMS and/or iTBS in MDD. For example, Baeken et al. [44] reported that positive connectivity between the sgACC and medial orbitofrontal cortex at baseline could differentiate responders from non-responders to accelerated iTBS. Another study reported that the higher FC within DMN and SN characterized responders for both iTBS and $10 \mathrm{~Hz}$ rTMS patient groups [45]. In the case of $5-\mathrm{Hz}$ rTMS, there are reports of more negative pretreatment FC between sgACC and DMN predicting clinical response [46]. For $10-\mathrm{Hz}$ rTMS, the connectivity of DLPFC to several brain regions, namely, reduced FC to the left caudate [47], higher FC to the striatum [48], and greater negative FC to the sgACC [39], has been shown to predict treatment response, highlighting the importance of not only DLPFC but also its FC to distant brain regions in the rTMS treatment mechanisms of MDD.

As is clear from above, there seems to be a wide variety of markers predicting rTMS and iTBS responses and symptoms improvement. In future studies, it would thus be important to replicate the predictive values and investigate combinations of markers that increase specificity and sensitivity or even promote the use of either rTMS or iTBS. Such future development of predictive features, possibly with multivariate data analysis, would allow for greater efficiency of rTMS in clinical settings, thereby benefitting the health care system and providing patient satisfaction by preventing treatments being undertaken that are not expected to work.

While rTMS has been established as an effective treatment for MDD, the percentage of patients that respond to this treatment remains low, varying from 40 to $50 \%$ [49]. This likely stems from interindividual variability in the response to rTMS. Some of the factors that contribute to these differences include age, gender, variations in 
Fig. 1. The standard MNI (anatomical) coordinate-based left DLPFC (denoted with a filled star) versus targets selected when employing individual rsfMRI data (denoted with filled circles).

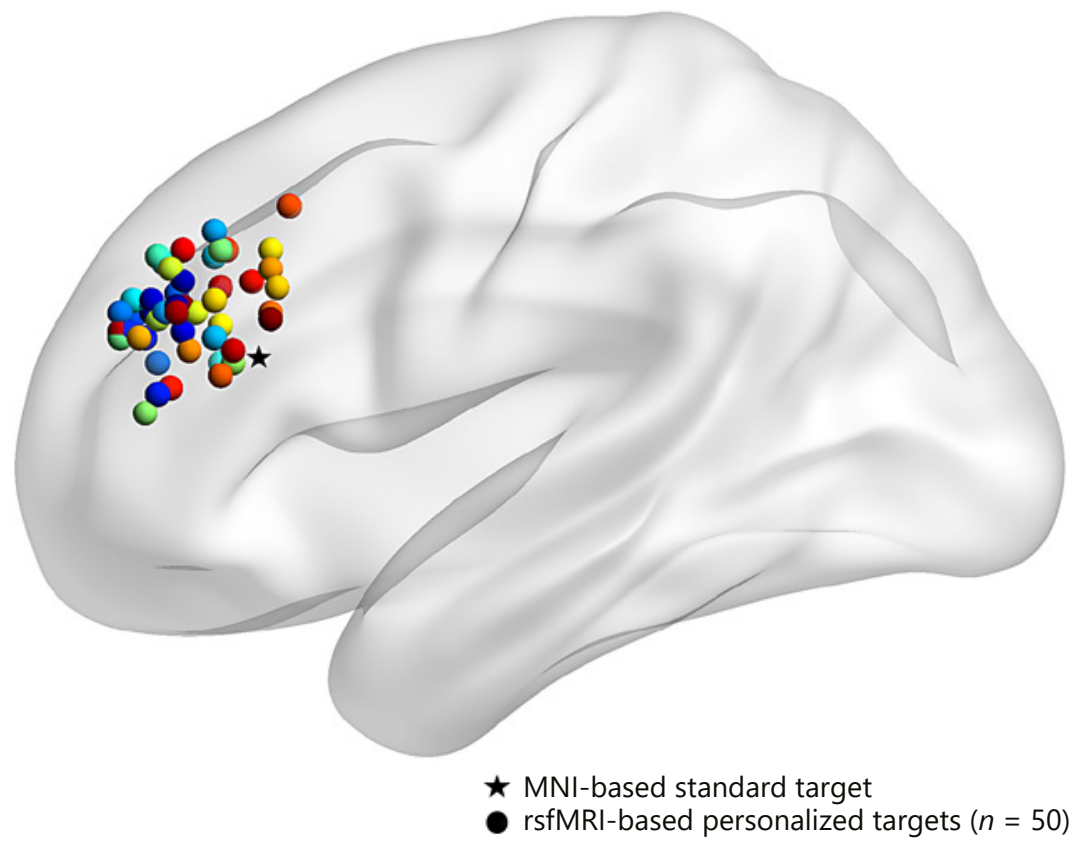

rsfMRI-based personalized targets $(n=50)$ individual cortical excitability, neurophysiological traits, white-matter connectivity, anatomical and functional variability, and genetic polymorphisms [16].

Although age has an influence on the effect of singleor paired-pulse techniques [50], evidence of rTMS being influenced by age remains inconclusive [16]. MEP variability, however, has been shown to be higher in females than in males [51]. White-matter connectivity also influences how the local effects of rTMS spread across networks, so a variation in white-matter pathways would contribute to differences in rTMS effects [52-54]. Similarly, as rTMS is known to cause its effects via tissue reorganization and plasticity (inducing a long-term potentiation/depression-like phenomenon), the genes involved in regulating such events can influence the outcome of rTMS $[55,56]$.

Other factors such as scalp-to-cortex distance can also influence the outcome, because the magnetic field induced by rTMS decays as a function of distance [27]. Hence, variations in scalp-to-cortex distance between subjects result in nonequivalent amounts of current being induced in the same cortical region when the same threshold, relative to RMT, is used for each individual. In this sense, simple corrective measures to calculate the re- quired compensatory increase or decrease in rTMS intensity based on scalp-to-cortex distance have been suggested [57].

Apart from the reasons mentioned above, the target site for stimulation is also important to note. In the case of rTMS for the treatment of depression, the common practice of delivering stimulation at $5 \mathrm{~cm}$ anterior to the motor cortex has been shown to be ineffective at reaching the desired DLPFC target [58]. Hence, it has been suggested that EEG-based landmarks and structural or functional MRI to guide the rTMS coil to the DLPFC using online neuronavigation systems be used. Figure 1 illustrates standard versus personalized sites for stimulation. However, an anatomically based landmark for targeting rTMS often fails to account for differences in the functional architecture of the brain from individual to individual [59-61]. Thus, using anatomical landmarks can provide a general location of various brain regions, but might still grossly miss the functionally important regions in terms of either network connectivity or functional activity.

We therefore suggest directing rTMS at cortical targets using functional activity-related information. This can be task-based fMRI, which uses a task that engages the re- 
gion of interest at which the stimulation is then aimed. The idea of engaging the aberrant targeted region arises from the knowledge that the effects of TMS or rTMS are very much dependent upon the activity occurring in the region being targeted $[62,63]$. Previous studies [64] have shown that TMS pulses or bursts usually activate the subpopulations of neurons, which are exhibiting the lowest levels of excitability. This is the state-dependency phenomenon of neurostimulation, where the current state of a subject's brain influences the outcome of the stimulation. Therefore, by engaging particular regions of the brain, researchers can probably influence the anticipated impact of rTMS.

Similarly, it can be based on rsfMRI, where networkbased FC is used to identify the important nodes of a network that are targeted using rTMS. As described in studies by Weigand et al. [39] and Fox et al. [40, 41] our research group utilized rsfMRI to identify left DLPFC targets based on the connectivity to the sgACC [65]. We selected rTMS target nodes in the left DLPFC that had a higher negative connectivity to the sgACC as it has been suggested that this has a better clinical outcome. Our study forms the basis that it is in fact practical to use individual rsfMRI to target rTMS, hence opening up new avenues for personalization of rTMS treatment.

However, fMRI sessions are currently not a standard part of MDD treatment and care, and the suggestion of personalized targeting of the site of stimulation based on FC data is a preliminary one that requires further validation. This fMRI-based decision could prove important for the $50 \%$ of patients who are refractory to therapy and so do not benefit from rTMS [49]. More RCTs exploring the benefits of FC-based versus anatomically based targeting (or 10/20 EEG-system based) are needed; this would encourage the clinical integration of fMRI data for use in rTMS therapy. If the efficacy of such an approach is established from multicenter RCTs, we believe the benefits will outweigh the costs associated with fMRI and would have a positive impact on tertiary health care of patients with MDD.

\section{Transcranial Direct Current Stimulation}

Low-intensity transcranial electrical stimulation (TES) methods encompass the external application of electrical current to the brain using at least two electrodes. The externally applied current modulates the spontaneous firing rates of neurons by de- or hyperpolarizing resting membrane potentials (as initially observed in the case of

Mechanisms and Challenges of

Neuromodulation by tDCS and rTMS
tDCS $>50$ years ago $[66,67])$, thereby changing cortical excitability and activity. Evaluating the functional and behavioral consequences of this method, low-intensity tDCS is particularly appropriate for gaining a further insight into the causative functional role of a given brain area and in functionally connected brain networks, e.g., how brain processes arise and could be changed. The magnitude and direction of the induced after effects are highly dependent on the duration and intensity of the stimulation as well as electrode size and montage [68]. Originally, the tDCS effect was estimated by measuring the amplitude of the MEPs induced by single-pulse TMS [69-71]. Several studies conducted on the M1 showed that the MEP size increased after approximately $10 \mathrm{~min}$ of anodal stimulation and decreased after cathodal stimulation [review 71] and remained in this state for up to 60 min after the end of the stimulation. Because this "longlasting" effect of tDCS is thought to be related to neuroplastic changes in the brain, many studies have addressed the issue of its impact on visual perception and cognitive functions, including motor-learning, working memory, and semantic and episodic memory in healthy subjects as well as its therapeutic applications in neurologic and neuropsychiatric diseases [e.g., 72-79].

In the last few years, studies have been published that question the efficacy of tDCS. High between- and withingroup variability and even individual variability were observed, and several of the studies could not be replicated by other investigators. The reason for the relatively high variability is far from being understood [80-85]. Many researchers and clinical practitioners concentrate on the manipulation of four adjustable tDCS parameters: current intensity, stimulation duration, electrode size, and electrode position. These technical variables are easily regulated and play a large role in $\mathrm{tDCS}$ effects. However, even these controllable parameters are not always discussed in any cogent manner with regard to increasing efficacy, understanding the mechanisms, or decreasing the variability.

The traditional description of tDCS effects is related to changes in "cortical excitability," i.e., it is thought that the anode that is positively charged enhances the excitability of the underlying cortex while a negatively charged cathode suppresses the excitability of the targeted cortical area [69]. Generally, there is evidence at the neuronal level that anodal tDCS hyperpolarizes the membrane potential in the apical dendritic regions and depolarizes it in the somatic region, whereas the cathodal electrode has an opposite effect [86-90]. Furthermore, besides cell morphology, the extent to which neurons are affected by tDCS 
(and how) also depends on the orientation of the cells with regard to the induced electric field.

TDCS is typically applied at $1-2 \mathrm{~mA}$ (maximum 4 $\mathrm{mA}$ ), but the electric field in the brain is reduced due to shunting effects (of skin and scalp); it is therefore estimated to maximally reach $0.4-0.8 \mathrm{mV} / \mathrm{mm}$ when $1.0 \mathrm{~mA}$ is applied externally $[91,92]$. While early studies observed that the magnitude and length of the induced after effects (at least after M1 stimulation) increased with higher current intensities and longer stimulation duration [70, 93], later studies reported that doubling the intensity of tDCS led to an opposite effect after cathodal stimulation [94] and increasing the duration of anodal tDCS to $26 \mathrm{~min}$ led to MEP decreases [95]. With regard to other cortical areas, the effects of different stimulation intensities and durations have not yet been systematically investigated.

The magnitude and duration of the after effects also depend on the functional state of the brain, i.e., whether the stimulation is given during rest or before/together with a motor or cognitive task [96]. When tDCS has no effect during tasks, it can be speculated that the effect is perhaps too weak to manifest when the activity is being performed [97].

The size of the electrodes and their montage are highly relevant for the efficacy of the stimulation. Conventionally, tDCS involves two electrodes placed on the scalp. Typical electrode sizes range between 4 and $35 \mathrm{~cm}^{2}$. A first limitation here derives from the wide electric field induced in the cortex by such large electrodes $[86,91,98$, 99]. The consequence is poor focusing, which can make the interpretation of the results difficult because of the impossibility of precisely locating the structures affected by tDCS. It should also be considered that almost all previous studies targeted single brain regions with low-intensity TES to modulate brain function. However, brain regions interact with each other through networks; the stimulation of a single brain area may thus influence and/ or be influenced by other regions and networks. Because of this complexity, the type of stimulation that was originally seen as "excitatory" (anodal tDCS) might not always increase "cortical excitability" and vice versa. A better description of the tDCS effect in the future might be that it modifies the "excitability-inhibitory balance" in the stimulated and related cortical areas. With regard to the parallel stimulation of multiple regions of a network [100], high-definition multielectrode tDCS arrays, of up to 32 electrodes (HD tDCS), have recently become available $[101,102]$.

Regarding the use of tDCS for the treatment of depression, the current approach is similar to that de- scribed above for rTMS. Most of the clinical trials have concentrated on enhancing the neural activity in the left DLPFC with anodal stimulation and/or reducing the neural activity in the right DLPFC with cathodal stimulation [11, 103-108]. Computer modeling and neuroimaging (fMRI) tDCS studies suggest that, in fact, the stimulation also largely affects deeper brain structures, such as the sgACC, amygdala, and hippocampus [86, 99, 109, 110].

The antidepressant effect of anodal tDCS on the left DLPFC was investigated in at least 15 RCTs [8], as well as in several case reports and open-labeled studies. Unfortunately, most of the RCTs investigating the beneficial effects of tDCS had large patient sample variability (related to the diagnosis of drug-resistant, unipolar, or bipolar depression) and different goals (comparing different stimulation protocols, add-on treatment [pharmacotherapy], or long-term treatment), so no solid conclusions can be made based on these data. One of the critical points is that the concomitant administration of antidepressant medication, benzodiazepines, and antiepileptics can influence tDCS-mediated effects on brain excitability and may indeed have increased variability and reduced the therapeutic impact in these studies. On the other hand, in a recent clinical trial, the combination of anodal tDCS with sertraline $(50 \mathrm{mg} /$ day) was superior to each treatment applied alone and to placebo, suggesting an additive interaction of tDCS and antidepressant medication (the SELECT-TDCS trial [111-113]). Previous studies implied that the outcome of $\mathrm{tDCS}$ in healthy subjects may be mediated by pharmacological modulation of noradrenergic serotonergic pathways. Therefore, serotonergic enhancement might increase the neuroplastic effects of anodal tDCS, thus resulting in synergistic effects [114, 115].

A precise understanding of the differences between responders and nonresponders may help in the identification of patients responsive to $\mathrm{tDCS}$ at the beginning of the therapy. In a recent trial (Escitalopram vs. Electrical Current Therapy for Treating Depression Clinical Study [ELECT-TDCS]), it was observed that the plasma levels of nerve growth factor predicted early depression improvement due to tDCS; this should be explored in further clinical studies [106].

Recent clinical guidelines recommend the following when using tDCS for treating depression: anodal tDCS of the left DLPFC (with right orbitofrontal cathode) delivered for at least 10 days (stimulation intensity: $2 \mathrm{~mA}$ and duration: $20-30 \mathrm{~min}$ ) in medicated or drug-free patients with MDD. There is also an appropriate amount of evi- 
Table 1. A summary of possible approaches for reducing variability in the rTMS and tDCS response in patients with depression

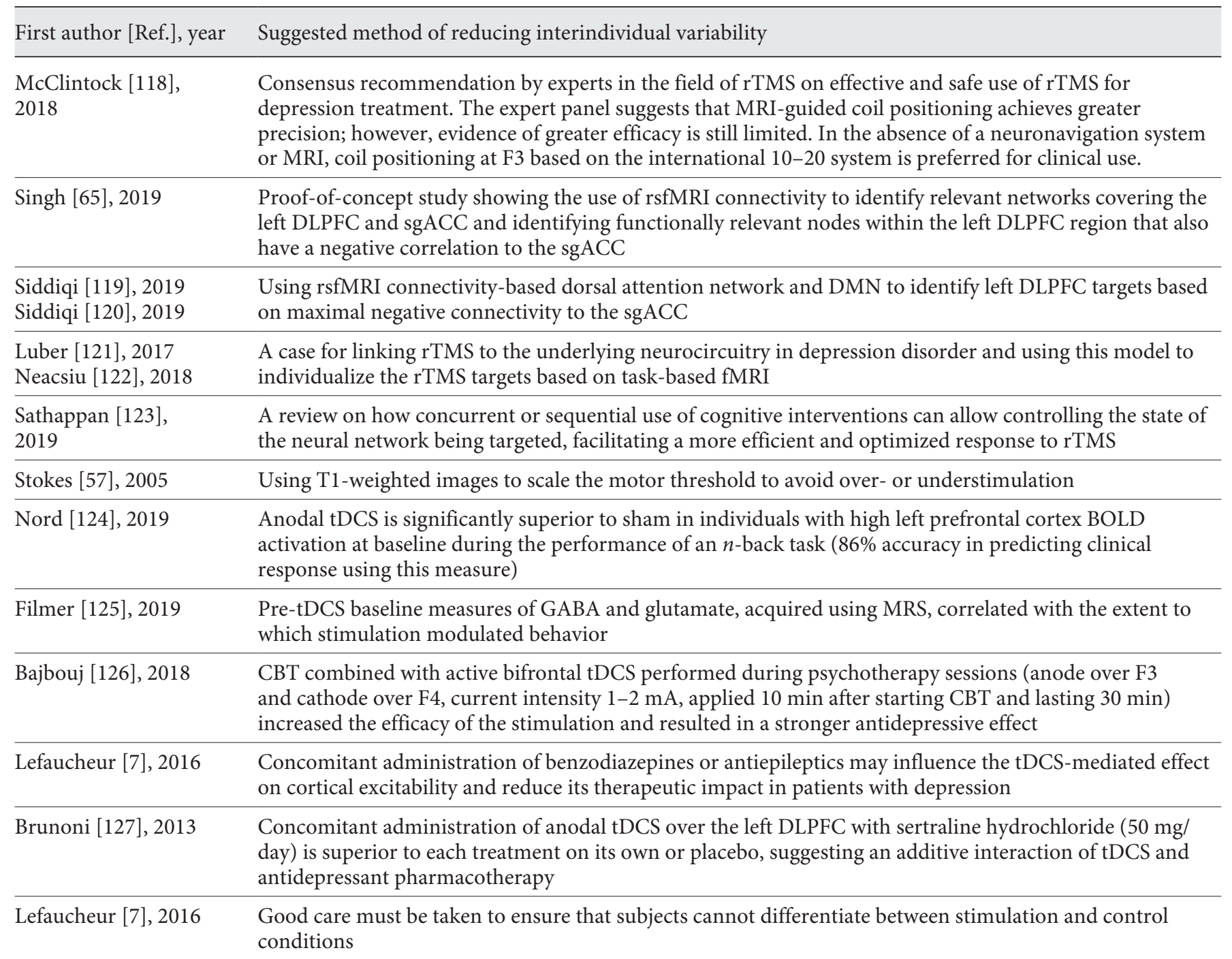

Of note, these suggestions may have the potential to be applied to other psychiatric disorders. Based on the underlying neural circuitry implicated in the psychopathology, neuroimaging methods can be adopted to more precisely target relevant brain regions/networks.

dence to make a level $\mathrm{B}$ recommendation concerning the absence of efficacy using the same tDCS protocol in patients with drug-resistant depression.

\section{Conclusion}

rTMS and tDCS have been widely explored for the treatment of depression and their efficacy, safety, and tolerability have been established. By combining these data with rsfMRI information, a developed FC-based model indicates that MDD is associated with aberrant DMN,
CEN, and SN connectivity, possibly due to hyperactivity of the sgACC. rTMS is believed to normalize these functional networks, which is reportedly in line with reduced sgACC activity in response to antidepressant treatment. Despite the current understanding, the effectiveness remains low for the treatment of MDD, partly stemming from age, gender, and individual genomic, anatomical, and functional variability. Of these, age and gender vary in the real-world patient population; an effort should thus be made to increase stimulation efficiency independent of age and gender. Table 1 summarizes suggestions to reduce variability in the response to standard rTMS by 
means of integrating individual neuroimaging data. As mentioned earlier, these recommendations are still in need of rigorous scientific testing. Multicenter RCTs will establish the utility of these approaches, for the treatment of depression and other psychiatric disorders.

Similar to rTMS, one of the critical points in tDCS is to increase the efficacy of stimulation. Early studies used a maximum of 20-min-long sessions of $1 \mathrm{~mA}$ anodal tDCS over the left DLPFC and a cathode placed over the right supraorbital region. Current trials support the use of a longer stimulation duration (30 min) and a higher intensity $(2 \mathrm{~mA})$, with the cathode placed over the right DLPFC. However, it must still be demonstrated whether increasing the duration and intensity of stimulation automatically leads to improved therapeutic efficacy in MDD. With regard to the long-term effects of tDCS, the number of studies is very limited. In a small study (on 11 patients) who completed a 3-month follow-up after a 10day $\mathrm{tDCS}$ protocol, it was found that $45 \%$ of the patients were still responders at the latest time point [116]. Nevertheless, a higher relapse rate has been reported when the repetition of the sessions was reduced from weekly to biweekly $[111,117]$.
Thus, we believe that attempting to reduce variability in factors such as target location and stimulation protocols, which contribute to the differences in response to brain stimulation, is the way forward for increasing the clinical effectiveness of transcranial stimulation. Furthermore, continued searches for predictive markers of brain stimulation response, molecular/genetic markers, and rsfMRI-based FC markers for both tDCS and rTMS will likely augment the effective use of brain stimulation. Adequately sensitive and specific markers will allow for more efficient use of both clinical resources, including better utilization of patients' time and the overall impact on health, thus benefiting the economy in the larger run.

\section{Acknowledgement}

A.S., T.E.-G., and R.G.-M. received support from the German Federal Ministry of Education (Bundesministerium für Bildung und Forschung, BMBF: 01 ZX 1507, "PreNeSt - e:Med").

\section{Disclosure Statement}

The authors declare there are no conflicts of interest.

\section{References}

1 Aleman A, Sommer IE, Kahn RS. Efficacy of slow repetitive transcranial magnetic stimulation in the treatment of resistant auditory hallucinations in schizophrenia: a meta-analysis. J Clin Psychiatry. 2007 Mar;68(3):416-21.

2 Devlin JT, Watkins KE. Stimulating language: insights from TMS. Brain. 2007 Mar; 130(Pt 3):610-22.

3 Fregni F, Pascual-Leone A. Technology insight: noninvasive brain stimulation in neurology-perspectives on the therapeutic potential of rTMS and tDCS. Nat Clin Pract Neurol. 2007 Jul;3(7):383-93.

4 George MS, Nahas Z, Borckardt JJ, Anderson B, Foust MJ, Burns C, et al. Brain stimulation for the treatment of psychiatric disorders. Curr Opin Psychiatry. 2007 May;20(3):250-4.

5 Levkovitz Y, Isserles M, Padberg F, Lisanby SH, Bystritsky A, Xia G, et al. Efficacy and safety of deep transcranial magnetic stimulation for major depression: a prospective multicenter randomized controlled trial. World Psychiatry. 2015 Feb;14(1):64-73.

6 Neef NE, Hoang TN, Neef A, Paulus W, Sommer M. Speech dynamics are coded in the left motor cortex in fluent speakers but not in adults who stutter. Brain. 2015 Mar;138(Pt 3): 712-25.

7 Lefaucheur JP. A comprehensive database of published tDCS clinical trials (2005-2016). Neurophysiol Clin. 2016 Dec;46(6):319-98.
8 Lefaucheur JP, Antal A, Ayache SS, Benninger $\mathrm{DH}$, Brunelin J, Cogiamanian F, et al. Evidence-based guidelines on the therapeutic use of transcranial direct current stimulation (tDCS). Clin Neurophysiol. 2017 Jan;128(1): 56-92.

9 Lefaucheur JP, André-Obadia N, Antal A, Ayache SS, Baeken C, Benninger DH, et al. Evidence-based guidelines on the therapeutic use of repetitive transcranial magnetic stimulation (rTMS). Clin Neurophysiol. 2014 Nov; 125(11):2150-206.

10 Brunoni AR, Valiengo L, Baccaro A, Zanao TA, de Oliveira JF, Vieira GP, et al. Sertraline vs. ELectrical Current Therapy for Treating Depression Clinical Trial-SELECT TDCS: design, rationale and objectives. Contemp Clin Trials. 2011 Jan;32(1):90-8.

11 Dondé C, Amad A, Nieto I, Brunoni AR, Neufeld NH, Bellivier F, et al. Transcranial direct-current stimulation (tDCS) for bipolar depression: A systematic review and metaanalysis. Prog Neuropsychopharmacol Biol Psychiatry. 2017 Aug;78:123-31.

12 Rossi S, Hallett M, Rossini PM, Pascual-Leone A, Avanzini G, Bestmann S, et al.; Safety of TMS Consensus Group. Safety, ethical considerations, and application guidelines for the use of transcranial magnetic stimulation in clinical practice and research. Clin Neurophysiol. 2009 Dec;120(12):2008-39.
13 Barker AT, Jalinous R, Freeston IL. Non-invasive magnetic stimulation of human motor cortex. Lancet. 1985 May;1(8437):1106-7.

14 Miranda PC. Physics of effects of transcranial brain stimulation. Handb Clin Neurol. 2013; 116:353-66.

15 Wagner T, Valero-Cabre A, Pascual-Leone A. Noninvasive human brain stimulation. Annu Rev Biomed Eng. 2007;9(1):527-65.

16 Valero-Cabré A, Amengual JL, Stengel C, Pascual-Leone A, Coubard OA. Transcranial magnetic stimulation in basic and clinical neuroscience: A comprehensive review of fundamental principles and novel insights. Neurosci Biobehav Rev. 2017 Dec;83(October):381-404.

17 Fitzgerald PB, Fountain S, Daskalakis ZJ. A comprehensive review of the effects of rTMS on motor cortical excitability and inhibition. Clin Neurophysiol. 2006 Dec;117(12):2584-96.

18 Mutz J, Edgcumbe DR, Brunoni AR, Fu CH. Efficacy and acceptability of non-invasive brain stimulation for the treatment of adult unipolar and bipolar depression: A systematic review and meta-analysis of randomised sham-controlled trials. Neurosci Biobehav Rev. 2018 Sep;92:291-303.

19 Simonetta-Moreau M. Non-invasive brain stimulation (NIBS) and motor recovery after stroke. Ann Phys Rehabil Med. 2014 Nov; 57(8):530-42. 
20 McGirr A, Berlim MT. Clinical Usefulness of Therapeutic Neuromodulation for Major Depression: A Systematic Meta-Review of Recent Meta-Analyses. Psychiatr Clin North Am. 2018 Sep;41(3):485-503.

21 Modugno N, Nakamura Y, MacKinnon CD, Filipovic SR, Bestmann S, Berardelli A, et al. Motor cortex excitability following short trains of repetitive magnetic stimuli. Exp Brain Res. 2001 Oct;140(4):453-9.

22 Halawa I, Goldental A, Shirota Y, Kanter I, Paulus W. Less might be more: conduction failure as a factor possibly limiting the efficacy of higher frequencies in rTMS protocols. Front Neurosci. 2018 May;12(MAY):358.

23 Klomjai W, Katz R, Lackmy-Vallée A. Basic principles of transcranial magnetic stimulation (TMS) and repetitive TMS (rTMS). Ann Phys Rehabil Med. 2015 Sep;58(4):208-13.

24 Philip NS, Barredo J, Aiken E, Carpenter LL. Neuroimaging Mechanisms of Therapeutic Transcranial Magnetic Stimulation for Major Depressive Disorder. Biol Psychiatry Cogn Neurosci Neuroimaging. 2018 Mar;3(3):21122.

25 Martinot JL, Hardy P, Feline A, Huret JD, Mazoyer B, Attar-Levy D, et al. Left prefrontal glucose hypometabolism in the depressed state: a confirmation. Am J Psychiatry. 1990 Oct;147(10):1313-7.

26 Speer AM, Kimbrell TA, Wassermann EM, D Repella J, Willis MW, Herscovitch P, et al. Opposite effects of high and low frequency rTMS on regional brain activity in depressed patients. Biol Psychiatry. 2000 Dec;48(12): 1133-41.

27 Nahas Z, Teneback CC, Kozel A, Speer AM, DeBrux C, Molloy M, et al. Brain effects of TMS delivered over prefrontal cortex in depressed adults: role of stimulation frequency and coil-cortex distance. J Neuropsychiatry Clin Neurosci. 2001;13(4):459-70.

28 Teneback CC, Speer AM, Stallings LE, Pharm $\mathrm{D}$, Spicer $\mathrm{KM}, \mathrm{Ph} \mathrm{D}$, et al. Changes in prefrontal cortex and paralimbic activity in depression following two weeks of daily left prefrontal TMS. J Neuropsychiatry Clin Neurosci. 1999;11(4):426-35.

29 Loo CK, Sachdev PS, Haindl W, Wen W, Mitchell PB, Croker VM, et al. High $(15 \mathrm{~Hz})$ and low $(1 \mathrm{~Hz})$ frequency transcranial magnetic stimulation have different acute effects on regional cerebral blood flow in depressed patients. Psychol Med. 2003 Aug;33(6):9971006.

30 George MS, Lisanby SH, Avery D, McDonald WM, Durkalski V, Pavlicova M, et al. Daily left prefrontal transcranial magnetic stimulation therapy for major depressive disorder: a sham-controlled randomized trial. Arch Gen Psychiatry. 2010 May;67(5):507-16.

31 O'Reardon JP, Solvason HB, Janicak PG, Sampson S, Isenberg KE, Nahas Z, et al. Efficacy and safety of transcranial magnetic stimulation in the acute treatment of major depression: a multisite randomized controlled trial. Biol Psychiatry. 2007 Dec;62(11):1208-16.
32 Berlim MT, Broadbent HJ, Van den Eynde F. Blinding integrity in randomized sham-controlled trials of repetitive transcranial magnetic stimulation for major depression: a systematic review and meta-analysis. Int J Neuropsychopharmacol. 2013 Jun;16(5):1173-81.

33 Zhang YQ, Zhu D, Zhou XY, Liu YY, Qin B, Ren GP, et al. Bilateral repetitive transcranial magnetic stimulation for treatment-resistant depression: a systematic review and metaanalysis of randomized controlled trials. Braz J Med Biol Res. 2015 Mar;48(3):198-206.

34 Huang YZ, Edwards MJ, Rounis E, Bhatia KP, Rothwell JC. Theta burst stimulation of the human motor cortex. Neuron. 2005 Jan; 45(2):201-6.

35 Blumberger DM, Vila-Rodriguez F, Thorpe KE, Feffer K, Noda Y, Giacobbe P, et al. Effectiveness of theta burst versus high-frequency repetitive transcranial magnetic stimulation in patients with depression (THREED): a randomised non-inferiority trial. Lancet. 2018 Apr;391(10131):1683-92.

36 Cooney RE, Joormann J, Eugène F, Dennis EL, Gotlib IH. Neural correlates of rumination in depression. Cogn Affect Behav Neurosci. 2010 Dec;10(4):470-8.

37 Liston C, Chen AC, Zebley BD, Drysdale AT, Gordon R, Leuchter B, et al. Default mode network mechanisms of transcranial magnetic stimulation in depression. Biol Psychiatry. 2014 Oct;76(7):517-26.

38 Goya-Maldonado R, Brodmann K, Keil M, Trost S, Dechent P, Gruber O. Differentiating unipolar and bipolar depression by alterations in large-scale brain networks. Hum Brain Mapp. 2016 Feb;37(2):808-18.

39 Weigand A, Horn A, Caballero R, Cooke D, Stern AP, Taylor SF, et al. Prospective Validation That Subgenual Connectivity Predicts Antidepressant Efficacy of Transcranial Magnetic Stimulation Sites. Biol Psychiatry. 2018 Jul;84(1):28-37.

40 Fox MD, Liu H, Pascual-Leone A. Identification of reproducible individualized targets for treatment of depression with TMS based on intrinsic connectivity. Neuroimage. 2013 Feb; 66:151-60

41 Fox MD, Buckner RL, White MP, Greicius $\mathrm{MD}$, Pascual-Leone A. Efficacy of transcranial magnetic stimulation targets for depression is related to intrinsic functional connectivity with the subgenual cingulate. Biol Psychiatry. 2012 Oct;72(7):595-603.

42 Mayberg HS, Lozano AM, Voon V, McNeely HE, Seminowicz D, Hamani C, et al. Deep brain stimulation for treatment-resistant depression. Neuron. 2005 Mar;45(5):651-60.

43 Drevets WC, Savitz J, Trimble M. The subgenual anterior cingulate cortex in mood disorders. CNS Spectr. 2008 Aug;13(8):663-81.

44 Baeken C, Duprat R, Wu GR, De Raedt R, van Heeringen K. Subgenual Anterior CingulateMedial Orbitofrontal Functional Connectivity in Medication-Resistant Major Depression: A Neurobiological Marker for Accelerated Intermittent Theta Burst Stimulation
Treatment? Biol Psychiatry Cogn Neurosci Neuroimaging. 2017 Oct;2(7):556-65.

45 Ge R, Blumberger DM, Downar J, Daskalakis ZJ, Dipinto AA, Tham JC, et al. Abnormal functional connectivity within resting-state networks is related to rTMS-based therapy effects of treatment resistant depression: A pilot study. J Affect Disord. 2017 Aug;218(6):75-81.

46 Philip NS, Barredo J, van 't Wout-Frank M, Tyrka AR, Price LH, Carpenter LL. Network Mechanisms of Clinical Response to Transcranial Magnetic Stimulation in Posttraumatic Stress Disorder and Major Depressive Disorder. Biol Psychiatry. 2018 Feb;83(3):26372.

47 Kang JI, Lee H, Jhung K, Kim KR, An SK, Yoon KJ, et al. Frontostriatal Connectivity Changes in Major Depressive Disorder After Repetitive Transcranial Magnetic Stimulation: A Randomized Sham-Controlled Study. J Clin Psychiatry. 2016 Sep;77(9):e1137-43.

48 Avissar M, Powell F, Ilieva I, Respino M, Gunning FM, Liston C, et al. Functional connectivity of the left DLPFC to striatum predicts treatment response of depression to TMS. Brain Stimul. 2017 Sep - Oct;10(5):919-25.

49 Bakker N, Shahab S, Giacobbe P, Blumberger DM, Daskalakis ZJ, Kennedy SH, et al. rTMS of the dorsomedial prefrontal cortex for major depression: safety, tolerability, effectiveness, and outcome predictors for $10 \mathrm{~Hz}$ versus intermittent theta-burst stimulation. Brain Stimul. 2015 Mar-Apr;8(2):208-15.

50 Peinemann A, Lehner C, Conrad B, Siebner HR. Age-related decrease in paired-pulse intracortical inhibition in the human primary motor cortex. Neurosci Lett. 2001 Nov;313(12):33-6.

51 Pitcher JB, Ogston KM, Miles TS. Age and sex differences in human motor cortex inputoutput characteristics. J Physiol. 2003 Jan; 546(Pt 2):605-13.

52 Quentin R, Chanes L, Migliaccio R, Valabrègue $\mathrm{R}$, Valero-Cabré A. Fronto-tectal white matter connectivity mediates facilitatory effects of non-invasive neurostimulation on visual detection. Neuroimage. 2013 Nov;82:344-54.

53 Quentin R, Chanes L, Vernet M, Valero-Cabré A. Fronto-Parietal Anatomical Connections Influence the Modulation of Conscious Visual Perception by High-Beta Frontal Oscillatory Activity. Cereb Cortex. 2015 Aug; 25(8):2095-101.

54 Quentin R, Elkin Frankston S, Vernet M, Toba MN, Bartolomeo P, Chanes L, et al. Visual Contrast Sensitivity Improvement by Right Frontal High-Beta Activity Is Mediated by Contrast Gain Mechanisms and Influenced by Fronto-Parietal White Matter Microstructure. Cereb Cortex. 2016 Jun;26(6): 2381-90.

55 Kleim JA, Chan S, Pringle E, Schallert K, Procaccio V, Jimenez R, et al. BDNF val66met polymorphism is associated with modified experience-dependent plasticity in human motor cortex. Nat Neurosci. 2006 Jun;9(6): 735-7. 
56 Cheeran B, Talelli P, Mori F, Koch G, Suppa A, Edwards M, et al. A common polymorphism in the brain-derived neurotrophic factor gene (BDNF) modulates human cortical plasticity and the response to rTMS. J Physiol. 2008 Dec;586(23):5717-25.

57 Stokes MG, Chambers CD, Gould IC, Henderson TR, Janko NE, Allen NB, et al. Simple metric for scaling motor threshold based on scalp-cortex distance: application to studies using transcranial magnetic stimulation. J Neurophysiol. 2005 Dec;94(6):4520-7.

58 Fitzgerald PB, Maller JJ, Hoy KE, Thomson R, Daskalakis ZJ. Exploring the optimal site for the localization of dorsolateral prefrontal cortex in brain stimulation experiments. Brain Stimul. 2009 Oct;2(4):234-7.

59 Dubois J, Adolphs R. Building a Science of Individual Differences from fMRI. Trends Cogn Sci. 2016 Jun;20(6):425-43.

60 Van Horn JD, Grafton ST, Miller MB. Individual Variability in Brain Activity: A Nuisance or an Opportunity? Brain Imaging Behav. 2008 Dec;2(4):327-34.

61 Miller MB, Van Horn JD. Individual variability in brain activations associated with episodic retrieval: a role for large-scale databases. Int J Psychophysiol. 2007 Feb;63(2):20513.

62 Silvanto J, Muggleton NG. New light through old windows: moving beyond the "virtual lesion" approach to transcranial magnetic stimulation. Neuroimage. 2008 Jan;39(2):549-52.

63 Silvanto J, Muggleton N, Walsh V. State-dependency in brain stimulation studies of perception and cognition. Trends Cogn Sci. 2008 Dec;12(12):447-54.

64 Silvanto J, Muggleton NG, Cowey A, Walsh V. Neural activation state determines behavioral susceptibility to modified theta burst transcranial magnetic stimulation. Eur J Neurosci. 2007 Jul;26(2):523-8.

65 Singh A, Erwin-Grabner T, Sutcliffe G, Antal A, Paulus W, Goya-Maldonado R. Personalized repetitive transcranial magnetic stimulation temporarily alters default mode network in healthy subjects. Sci Rep. 2019 Apr;9(1): 5631.

66 Creutzfeldt OD, Fromm GH, Kapp H. Influence of transcortical d-c currents on cortical neuronal activity. Exp Neurol. 1962 Jun;5(6): 436-52.

67 Bindman LJ, Lippold OC, Redfearn JW. The action of brief polarizing currents on the cerebral cortex of the rat (1) during current flow and (2) in the production of long-lasting after-effects. J Physiol. 1964 Aug;172(3):36982.

68 Woods AJ, Antal A, Bikson M, Boggio PS, Brunoni AR, Celnik P, et al. A technical guide to $\mathrm{tDCS}$, and related non-invasive brain stimulation tools. Clin Neurophysiol. 2016 Feb; 127(2):1031-48.

69 Nitsche MA, Paulus W. Excitability changes induced in the human motor cortex by weak transcranial direct current stimulation. J Physiol. 2000 Sep;527(Pt 3):633-9.
70 Nitsche MA, Paulus W. Sustained excitability elevations induced by transcranial DC motor cortex stimulation in humans. Neurology. 2001 Nov;57(10):1899-901.

71 Nitsche MA, Paulus W. Transcranial direct current stimulation-update 2011. Restor Neurol Neurosci. 2011;29(6):463-92.

72 Been G, Ngo TT, Miller SM, Fitzgerald PB, editors. The use of tDCS and CVS as methods of non-invasive brain stimulation. In: Brain Research Reviews, Vol. 56. Elsevier; 2007. pp. 346-61.

73 Doruk D, Gray Z, Bravo GL, Pascual-Leone A, Fregni F. Effects of tDCS on executive function in Parkinson's disease. Neurosci Lett. 2014 Oct;582:27-31.

74 Flöel A. tDCS-enhanced motor and cognitive function in neurological diseases. Neuroimage. 2014 Jan;85(Pt 3):934-47.

75 Hsu WY, Ku Y, Zanto TP, Gazzaley A. Effects of noninvasive brain stimulation on cognitive function in healthy aging and Alzheimer's disease: a systematic review and meta-analysis. Neurobiol Aging. 2015 Aug;36(8):234859.

76 Jacobson L, Koslowsky M, Lavidor M. tDCS polarity effects in motor and cognitive domains: a meta-analytical review. Exp Brain Res. 2012 Jan;216(1):1-10.

77 Tremblay S, Lepage JF, Latulipe-Loiselle A, Fregni F, Pascual-Leone A, Théoret $\mathrm{H}$. The uncertain outcome of prefrontal tDCS. Brain Stimul. 2014 Nov-Dec;7(6):773-83.

78 Prehn K, Flöel A. Potentials and limits to enhance cognitive functions in healthy and pathological aging by tDCS. Front Cell Neurosci. 2015 Sep;9:355.

79 Roe JM, Nesheim M, Mathiesen NC, Moberget T, Alnæs D, Sneve MH. The effects of tDCS upon sustained visual attention are dependent on cognitive load. Neuropsychologia. 2016 Jan;80:1-8.

80 Horvath JC, Vogrin SJ, Carter O, Cook MJ, Forte JD. Effects of a common transcranial direct current stimulation (tDCS) protocol on motor evoked potentials found to be highly variable within individuals over 9 testing sessions. Exp Brain Res. 2016 Sep;234(9):262942.

81 Jung-Hoon Kim. Do-Won Kim, Won-Hyuk Chang, Yun-Hee Kim, Chang-Hwan Im. Inconsistent outcomes of transcranial direct current stimulation (tDCS) may be originated from the anatomical differences among individuals: a simulation study using individual MRI data. In: 2013 35th Annual International Conference of the IEEE Engineering in Medicine and Biology Society (EMBC). IEEE; 2013. p. 823-5.

82 Laakso I, Tanaka S, Koyama S, De Santis V, Hirata A. Inter-subject Variability in Electric Fields of Motor Cortical tDCS. Brain Stimul. 2015 Sep-Oct; 8(5):906-13.

83 Madhavan S, Sriraman A, Freels S, Madhavan S, Sriraman A, Freels S. Reliability and Variability of $\mathrm{tDCS}$ Induced Changes in the Lower Limb Motor Cortex. Brain Sci. 2016 Jul;6(3):26.
84 Buch ER, Santarnecchi E, Antal A, Born J, Celnik PA, Classen J, et al. Effects of tDCS on motor learning and memory formation: $\mathrm{A}$ consensus and critical position paper. Clin Neurophysiol. 2017 Apr;128(4):589-603.

85 Wörsching J, Padberg F, Helbich K, Hasan A, Koch L, Goerigk S, et al. Test-retest reliability of prefrontal transcranial Direct Current Stimulation (tDCS) effects on functional MRI connectivity in healthy subjects. Neuroimage. 2017 Jul;155:187-201.

86 Bikson M, Rahman A, Datta A. Computational models of transcranial direct current stimulation. Clin EEG Neurosci. 2012 Jul;43(3): 176-83.

87 Abdel Rahman AM, Ryczko M, Pawling J, Dennis JW. Probing the hexosamine biosynthetic pathway in human tumor cells by multitargeted tandem mass spectrometry. ACS Chem Biol. 2013 Sep;8(9):2053-62.

88 Bikson M, Name A, Rahman A. Origins of specificity during tDCS: anatomical, activityselective, and input-bias mechanisms. Front Hum Neurosci. 2013 Oct;7:688.

89 Rahman A, Reato D, Arlotti M, Gasca F, Datta A, Parra LC, et al. Cellular effects of acute direct current stimulation: somatic and synaptic terminal effects. J Physiol. 2013 May; 591(10):2563-78.

90 Rahman A, Toshev PK, Bikson M. Polarizing cerebellar neurons with transcranial Direct Current Stimulation. Clin Neurophysiol. 2014 Mar;125(3):435-8.

91 Datta A, Bansal V, Diaz J, Patel J, Reato D, Bikson M. Gyri-precise head model of transcranial direct current stimulation: improved spatial focality using a ring electrode versus conventional rectangular pad. Brain Stimul. 2009 Oct;2(4):201-7.

92 Radman T, Datta A, Ramos RL, Brumberg JC Bikson M. One-dimensional representation of a neuron in a uniform electric field. In: 2009 Annual International Conference of the IEEE Engineering in Medicine and Biology Society. IEEE; 2009. p. 6481-4.

93 Nitsche MA, Nitsche MS, Klein CC, Tergau F, Rothwell JC, Paulus W. Level of action of cathodal DC polarisation induced inhibition of the human motor cortex. Clin Neurophysiol. 2003 Apr;114(4):600-4.

94 Batsikadze G, Moliadze V, Paulus W, Kuo MF, Nitsche MA. Partially non-linear stimulation intensity-dependent effects of direct current stimulation on motor cortex excitability in humans. J Physiol. 2013 Apr;591(7): 1987-2000.

95 Monte-Silva K, Kuo MF, Hessenthaler S, Fresnoza S, Liebetanz D, Paulus W, et al. Induction of late LTP-like plasticity in the human motor cortex by repeated non-invasive brain stimulation. Brain Stimul. 2013 May; 6(3):424-32.

96 Antal A, Terney D, Poreisz C, Paulus W. Towards unravelling task-related modulations of neuroplastic changes induced in the human motor cortex. Eur J Neurosci. 2007 Nov; 26(9):2687-91. 
97 Paulus W, Rothwell JC. Membrane resistance and shunting inhibition: where biophysics meets state-dependent human neurophysiology. J Physiol. 2016 May;594(10): 2719-28.

98 Bikson M, Datta A, Rahman A, Scaturro J. Electrode montages for tDCS and weak transcranial electrical stimulation: role of "return" electrode's position and size. Clin Neurophysiol. 2010 Dec;121(12):1976-8.

99 Bikson M, Datta A. Guidelines for precise and accurate computational models of tDCS. Brain Stimul. 2012 Jul;5(3):430-1.

100 Fischer DB, Fried PJ, Ruffini G, Ripolles O, Salvador R, Banus J, et al. Multifocal tDCS targeting the resting state motor network increases cortical excitability beyond traditional tDCS targeting unilateral motor cortex. Neuroimage. 2017 Aug;157:34-44.

101 Villamar MF, Volz MS, Bikson M, Datta A, Dasilva AF, Fregni F. Technique and considerations in the use of $4 \times 1$ ring high-definition transcranial direct current stimulation (HD-tDCS). J Vis Exp. 2013 Jul;(77):e50309.

102 Alam M, Truong DQ, Khadka N, Bikson M. Spatial and polarity precision of concentric high-definition transcranial direct current stimulation (HD-tDCS). Phys Med Biol. 2016 Jun;61(12):4506-21.

103 Bai S, Dokos S, Ho KA, Loo C. A computational modelling study of transcranial direct current stimulation montages used in depression. Neuroimage. 2014 Feb;87:332-44.

104 Palm U, Hasan A, Strube W, Padberg F. tDCS for the treatment of depression: a comprehensive review. Eur Arch Psychiatry Clin Neurosci. 2016 Dec;266(8):681-94.

105 Brunoni AR, Moffa AH, Sampaio-Junior B, Borrione L, Moreno ML, Fernandes RA, et al.; ELECT-TDCS Investigators. Trial of Electrical Direct-Current Therapy versus Escitalopram for Depression. N Engl J Med. 2017 Jun;376(26):2523-33.

106 Brunoni AR, Padberg F, Vieira EL, Teixeira AL, Carvalho AF, Lotufo PA, et al. Plasma biomarkers in a placebo-controlled trial comparing tDCS and escitalopram efficacy in major depression. Prog Neuropsychopharmacol Biol Psychiatry. 2018 Aug;86: 211-7.

107 Sampaio-Junior B, Tortella G, Borrione L, Moffa AH, Machado-Vieira R, Cretaz E, et al. Efficacy and Safety of Transcranial Direct Current Stimulation as an Add-on Treatment for Bipolar Depression: A Randomized Clinical Trial. JAMA Psychiatry. 2018 Feb;75(2):158-66

108 Welch ES, Weigand A, Hooker JE, Philip NS, Tyrka AR, Press DZ, et al. Feasibility of Computerized Cognitive-Behavioral Therapy Combined With Bifrontal Transcranial Direct Current Stimulation for Treatment of Major Depression. Neuromodulation. 2018. DOI: $10.1111 /$ ner.12807.
109 Keeser D, Meindl T, Bor J, Palm U, Pogarell $\mathrm{O}$, Mulert C, et al. Prefrontal transcranial direct current stimulation changes connectivity of resting-state networks during fMRI. J Neurosci. 2011 Oct;31(43):15284-93.

110 Keeser D, Padberg F, Reisinger E, Pogarell O, Kirsch V, Palm U, et al. Prefrontal direct current stimulation modulates resting EEG and event-related potentials in healthy subjects: a standardized low resolution tomography (sLORETA) study. Neuroimage. 2011 Mar;55(2):644-57.

111 Valiengo L, Benseñor IM, Goulart AC, de Oliveira JF, Zanao TA, Boggio PS, et al. The sertraline versus electrical current therapy for treating depression clinical study (selectTDCS): results of the crossover and followup phases. Depress Anxiety. 2013 Jul;30(7): 646-53.

112 Brunoni AR, Machado-Vieira R, SampaioJunior B, Vieira EL, Valiengo L, Benseñor IM, et al. Plasma levels of soluble TNF receptors 1 and 2 after tDCS and sertraline treatment in major depression: results from the SELECT-TDCS trial. J Affect Disord. 2015 Oct;185:209-13.

113 Brunoni AR, Tortella G, Benseñor IM Lotufo PA, Carvalho AF, Fregni F. Cognitive effects of transcranial direct current stimulation in depression: results from the SELECT-TDCS trial and insights for further clinical trials. J Affect Disord. 2016 Sep;202: 46-52.

114 Nitsche MA, Kuo MF, Karrasch R, Wächter B, Liebetanz D, Paulus W. Serotonin affects transcranial direct current-induced neuroplasticity in humans. Biol Psychiatry. 2009 Sep;66(5):503-8.

115 Kuo HI, Paulus W, Batsikadze G, Jamil A, Kuo MF, Nitsche MA. Chronic Enhancement of Serotonin Facilitates Excitatory Transcranial Direct Current StimulationInduced Neuroplasticity. Neuropsychopharmacology. 2016 Apr;41(5):1223-30.

116 Dell'Osso B, Dobrea C, Arici C, Benatti B, Ferrucci R, Vergari M, et al. Augmentative transcranial direct current stimulation (tDCS) in poor responder depressed patients: a follow-up study. CNS Spectr. 2014 Aug;19(4):347-54.

117 Martin DM, Alonzo A, Ho KA, Player M, Mitchell PB, Sachdev P, et al. Continuation transcranial direct current stimulation for the prevention of relapse in major depression. J Affect Disord. 2013 Jan;144(3):274-8.

118 McClintock SM, Reti IM, Carpenter LL, McDonald WM, Dubin M, Taylor SF, et al.; National Network of Depression Centers rTMS Task Group; American Psychiatric Association Council on Research Task Force on Novel Biomarkers and Treatments. Consensus Recommendations for the Clinical Application of Repetitive Transcranial Magnetic Stimulation (rTMS) in the Treatment of Depression. J Clin Psychiatry. 2018 Jan/Feb; 79(1):35-48.
119 Siddiqi SH, Trapp NT, Shahim P, Hacker CD, Laumann TO, Kandala S, et al. Individualized Connectome-Targeted Transcranial Magnetic Stimulation for Neuropsychiatric Sequelae of Repetitive Traumatic Brain Injury in a Retired NFL Player. J Neuropsychiatry Clin Neurosci. 2019. DOI: 10.1176/ appi.neuropsych.18100230.

120 Siddiqi SH, Trapp NT, Hacker CD, Laumann TO, Kandala S, Hong X, et al. Repetitive Transcranial Magnetic Stimulation with Resting-State Network Targeting for Treatment-Resistant Depression in Traumatic Brain Injury: A Randomized, Controlled, Double-Blinded Pilot Study. J Neurotrauma. 2019 Apr;36(8):1361-74.

121 Luber BM, Davis S, Bernhardt E, Neacsiu A, Kwapil L, Lisanby SH, et al. Using neuroimaging to individualize TMS treatment for depression: toward a new paradigm for imaging-guided intervention. Neuroimage. 2017 Mar;148:1-7.

122 Neacsiu AD, Luber BM, Davis SW, Bernhardt E, Strauman TJ, Lisanby SH. On the Concurrent Use of Self-System Therapy and Functional Magnetic Resonance ImagingGuided Transcranial Magnetic Stimulation as Treatment for Depression. J ECT. 2018 Dec;34(4):266-73.

123 Sathappan AV, Luber BM, Lisanby SH. The Dynamic Duo: combining noninvasive brain stimulation with cognitive interventions. Prog Neuropsychopharmacol Biol Psychiatry. 2019 Mar;89:347-60.

124 Nord CL, Halahakoon DC, Limbachya T, Charpentier C, Lally N, Walsh V, et al. Neural predictors of treatment response to brain stimulation and psychological therapy in depression: a double-blind randomized controlled trial. Neuropsychopharmacology. 2019 Aug;44(9):1613-22.

125 Filmer HL, Ehrhardt SE, Bollmann S, Mattingley JB, Dux PE. Accounting for individual differences in the response to tDCS with baseline levels of neurochemical excitability. Cortex. 2019 Jun;115:324-34.

126 Bajbouj M, Aust S, Spies J, Herrera-Melendez AL, Mayer SV, Peters M, et al. PsychotherapyPlus: augmentation of cognitive behavioral therapy (CBT) with prefrontal transcranial direct current stimulation (tDCS) in major depressive disorder-study design and methodology of a multicenter double-blind randomized placebo-controlled trial. Eur Arch Psychiatry Clin Neurosci. 2018 Dec;268(8):797-808.

127 Brunoni AR, Valiengo L, Baccaro A, Zanão TA, de Oliveira JF, Goulart A, et al. The sertraline vs. electrical current therapy for treating depression clinical study: results from a factorial, randomized, controlled trial. JAMA Psychiatry. 2013 Apr;70(4):38391.
Mechanisms and Challenges of Neuromodulation by tDCS and rTMS
Neuropsychobiology 2020;79:397-407 DOI: $10.1159 / 000502149$ 